\title{
Response to "Severe pernicious anemia with distinct cytogenetic and flow cytometric aberrations mimicking myelodysplastic syndrome"
}

\author{
Denise A. Wells • Michael R. Loken
}

Received: 18 May 2012 / Accepted: 1 June 2012 / Published online: 16 June 2012

(C) Springer-Verlag 2012

\section{Dear Editor,}

In a previous letter "Severe pernicious anemia with distinct cytogenetic and flow cytometric aberrations mimicking myelodysplastic syndrome" [1], the authors state that in using the established flow cytometric scoring system (FCM) from our manuscript by $\mathrm{Chu}$ et al. [2], a case of vitamin $\mathrm{B}_{12}$ deficiency was suspicious for myelodysplastic syndrome by showing an abnormal CD13/CD11b pattern.

We respectfully disagree with the following points:

1. The FCM, validated in multiple studies, $[3,4]$ did not use the reagent combination described: the exact antibody combinations and relationships as published are the only studies validated and, therefore, the only correct context of use for FCM.

2. If the abnormal CD13/CD11b were an isolated (single) abnormality, the FCM would be 1, with a sensitivity of $84 \%$ (stated in the Chu's manuscript), and in the original paper by Wells et al., a score of 1 was described as below the cutoff for myelodysplastic syndrome (MDS) when compared to non-MDS controls.

(a) The letter erroneously states that an FCM score of 1 was described in our validated studies as an "intermediate risk."

(b) The paper by Chu et al. stated that single abnormalities are occasionally observed in the CD13/ CD16 relationship in vitamin $\mathrm{B}_{12}$ deficiency anemia.

D. A. Wells $(\bowtie) \cdot$ M. R. Loken

Hematologics, Inc.,

3161 Elliot Ave. N.,

Seattle, WA 98211, USA

e-mail: dwellsmd@hematologics.com
In conclusion, based on utilization of the FCM as validated, we do not concur that there is evidence by flow cytometry from the data provided to make a diagnosis or suspicion of MDS in this case. The karyotyping results are concerning for an underlying dysplasia that is now masked by recovering hematopoiesis and additional follow-up is warranted.

Denise A. Wells, M.D.

Michael R. Loken, Ph.D.

Hematologics, Inc.

\section{References}

1. Parmentier S, Meinel J, Oelschlaegel U, Mohr B, Ehninger G, Schaich M, Platzbecker U (2012) Severe pernicious anemia with distinct cytogenetic and flow cytometric aberrations mimicking myelodysplastic syndrome. Ann Hematol. doi:10.1007/s00277012-1488-0

2. Chu SC, Wang TF, Li CC, Kao RH, Li DK, Su YC, Wells DA, Loken MR (2011) Flow cytometric scoring system as a diagnostic and prognostic tool in myelodysplastic syndromes. Leuk Res 35:868-873

3. Wells DA, Benesch M, Loken MR, Vallejo C, Myerson D, Leisenring WM, Deeg HJ (2003) Myeloid and monocytic dyspoiesis as determined by flow cytometric scoring in myelodysplastic syndrome correlates with the IPSS and with outcome after hematopoietic stem cell transplantation. Blood 102(1):394-403. doi:10.1182/ blood-2002-09-2768

4. Scott BL, Wells DA, Loken MR, Myerson D, Leisenring WM, Deeg HJ (2008) Validation of a flow cytometric scoring system as a prognostic indicator for posttransplantation outcome in patients with myelodysplastic syndrome. Blood 112(7):2681-2686. doi:10.1182/ blood-2008-05-153700 